\title{
Editorial: Spores and Spore Formers
}

\author{
Imrich Barák* \\ Department of Microbial Genetics, Institute of Molecular Biology, Slovak Academy of Sciences, Bratislava, Slovakia
}

Keywords: bacterial spores, Bacillus subtilis, Clostridia, initiation of sporulation, germination and dormancy, regulation of sporulation

\section{Editorial on the Research Topic}

\section{Spores and Spore Formers}

Bacterial spore formers havebeen the focus of intense study for almost half a century. The most heavily studied of these is Bacillus subtilis, an internationally recognized model organism, whose physiology, biochemistry, and genetics have been studied for many years. Under nutrient rich conditions, B. subtilis grows and multiplies by a process of cell expansion followed by division at mid-cell to generate identical daughter cells; however, B. subtilis also has the ability to form spores, dormant cells which are resistant to many of the chemical and physical challenges that normally kill bacteria. Although many of the basic aspects of this process are now well-understood, bacterial sporulation still remains a highly attractive model for studying various cell processes at a molecular level. There are several reasons for this interest. First, some of the more complex sporulation steps are not fully understood or are only described using "controversial" models.

OPEN ACCESS

Edited by:

Michael Sauer,

University of Natural Resources and Life Sciences, Vienna, Austria

Reviewed by:

Michael Sauer

University of Natural Resources and

Life Sciences, Vienna, Austria

Paola Branduardi,

University of Milano-Bicocca, Italy

${ }^{*}$ Correspondence:

Imrich Barák

imrich.barak@savba.sk

Specialty section

This article was submitted to Microbial Physiology and Metabolism,

a section of the journal

Frontiers in Microbiology

Received: 11 January 2017

Accepted: 24 May 2017

Published: 08 June 2017

Citation:

Barák I (2017) Editorial: Spores and

Spore Formers.

Front. Microbiol. 8:1046.

doi: 10.3389/fmicb.2017.01046
Second, the extensive attention lavished on the unicellular development of $B$. subtilis, a single microorganism, has left us largely ignorant of the multitude of different sporulation mechanisms present in many other Gram-positive endospore and exospore formers; this diversity would likely increase if the sporulation processes of the Gram-negative spore formers were also included. The ability of spores to lie dormant and then germinate presents both threats and potential benefits to human health and welfare. Botulism and tetanus are infectious diseases transmitted by spores, while the spores of Clostridium difficile are responsible for hospital-acquired infections, which are harmful to patients and expensive to treat and eradicate. Bacillus cereus spores cause food poisoning and present a challenge to the food industry, while the spores of B. anthracis, the cause of anthrax, are a concern because of their potential use as bioterrorism and biowarfare agents. On the other hand, spore formers also have great potential in applied research. Spore forming bacteria are becoming increasingly important in the areas of probiotics, vaccine technology, and biotechnology. This Research Topic in Frontiers in Microbiology details the most recent advances in spore research basic science, covers emerging areas of scientific importance involving the use of spores, including their use as probiotics in humans and animals, and examines the use of spores as tools for nanobiotechnology where the spore surface can be used to efficiently display heterologous proteins. In addition, this Topic covers the ecological roles of spores, the taxonomy and systematics of spore forming bacteria, and the architecture and assembly of spores. This Research Topic was partially but not exclusively linked to the 7th European Spores Conference, held in Egham (UK) on 18-20 April 2016 (http://sporesconference.org/).

Generally, the sporulation process can be thought of as the last opportunity for the bacterial cell to endure when all other attempts to grow, compete, and survive have been exhausted. Sporulation initiation is a complex process in which different types of input, including nutritional, population density, and cell cycle signals, are integrated by the cell before the decision to sporulate is made. Sophisticated and sensitive signal transduction pathways are essential for accurately monitoring all these signals. In B. subtilis and most endospore-forming bacteria, sporulation initiation is under the control of a phosphorelay, an expanded two-component sensory signaling system, and the key 
molecule in transducing signals into the activation of those genes required for sporulation is the response regulator Spo0A. In this Research Topic, Lecca et al. analyse numerical simulations of a model of the gene network which regulates sporulation initiation in Bacillus subtilis to find that the non-linearity underlying the time series data is due to low-dimensional chaos. Pompeo et al. review the importance of serine/threonine protein kinases in sporulation regulation in $B$. subtilis.

The sporulation process is controlled at the gene expression level by a complex regulatory cascade composed of different compartment-specific sigma factors and additional regulator proteins, which are highly conserved among various spore formers. Despite this conservation, both regulatory and phenotypic differences are observed between different species of spore forming bacteria. Eijlander et al., demonstrate that deletion of the regulatory sporulation protein SpoVT results in a severe sporulation defect in B. cereus, but not in B. subtilis.

The resistance of spores to environmental attacks is attributable to the unique morphology of the spore, which is formed by several layers: the core, the cortex, the coat, the crust, and, in some cases, the exosporium. Dipicolinic acid (DPA), with its ability to bind $\mathrm{Ca}^{2+}$ ions, plays a key role in the dehydration and mineralization of the spore core. Dehydration of the spore core is the main factor underlying the resistance of spores to wet heat. The work of Jamroskovic et al. cataloged the heterogeneity in the heat resistance capacity and the overall DPA/calcium content in the spores of several Clostridium species. Edwards et al. characterized the chemical and stress resistances of $C$. difficile spores. Abhyankar et al. determined the influence of sporulation conditions on spore coat composition in B. subtilis. The spores of some Bacillus species are surrounded by the exosporium, an outermost surface layer. Lanzilli et al. clarified the B. megaterium exosporium permeability.

Under favorable conditions, spores germinate to produce vegetative cells that multiply. Brunt et al. characterized the diversity of germination apparatuses in different C. botulinum groups. Nagler et al. identified genes which are differentially expressed during $B$. subtilis germination in high-salinity environments. Besides sporulation, $B$. cereus can also undergo a differentiation process in which short swimmer cells become elongated and hyperflagellated swarmer cells which favor the migration of the bacterial community on a surface. Mazzantini et al. showed that the flagellar FlhF protein is required for the swarming motility and full pathogenicity of this bacterium.

\section{AUTHOR CONTRIBUTIONS}

The author confirms being the sole contributor of this work and approved it for publication.

\section{ACKNOWLEDGMENTS}

The author receives support from the Slovak Academy of Sciences (Grant 2/0007/17), from the Slovak Research and Development Agency under contract APVV-14-0181, and from FNSNF (SCOPES IZ 73Z0_152527/1). The author thanks to Research Topic editors and organizers of 7th European Spores Conference-Simon Cutting, Ezio Ricca, Neil Fairweather, and Ivan Mijakovic. He also thanks to Jacob Bauer for helpful comments.

Conflict of Interest Statement: The author declares that the research was conducted in the absence of any commercial or financial relationships that could be construed as a potential conflict of interest.

Copyright () 2017 Barák. This is an open-access article distributed under the terms of the Creative Commons Attribution License (CC BY). The use, distribution or reproduction in other forums is permitted, provided the original author(s) or licensor are credited and that the original publication in this journal is cited, in accordance with accepted academic practice. No use, distribution or reproduction is permitted which does not comply with these terms. 